\title{
Pathological Aspects of Endometriosis
}

\author{
Agatha Kondi-Pafitis \\ Aretaieion University Hospital Athens Medical School,
}

Greece

\section{Introduction}

Endometriosis is one of the most enigmatic diseases of the female because its cause and pathophysiology remain poorly understood. Although the histological diagnosis of endometriosis is usually easy to make, there are diagnostic problems in relation to certain aspects such as the atypias and neoplastic alterations of the endometriotic glandular and stromal components and in cases of endometriosis in unusual places the small size of specimens available for histological examination does not aid the correct diagnosis. An effort will be made in this chapter to address the various problems and the most commonly asked questions about endometriosis.

\section{Definition of endometriosis}

Endometriosis is the presence of tissue resembling endometrial components outside the uterus. Usually both, glands and stroma are observed but occasionally the diagnosis can be made when only one component is present. Endometriosis is distinct from adenomyosis or endometriosis interna which is defined by the presence of endometrial components in the myometrium. These two entities were linked in the past by common terminology but present different clinical presentation, epidemiologic and etiologic patterns.

\section{Clinical features of endometriosis}

Endometriosis is a condition described in European Medical history for over 300 years (Knapp , 1999) and afflicts women in their reproductive years. It is the third leading cause of gynecologic hospitalization in the USA and although the true incidence is unknown, it is estimated that it afflicts about $4-13 \%$ of all women in reproductive age, $25-50 \%$ of women with infertility problems, $5-25 \%$ of those that are hospitalized because of pelvic pain, $50 \%$ of young girls with severe dysmenorrhea and up to $7 \%$ of women hospitalized with the diagnosis of pelvic masses(Cramer \& Missmer, 2002, Allaire, 2006, Anaf et al, 2000).There is a shift of the time of diagnosis from the late thirties and early forties to the twenties and the cause of this my be extensive use of laparoscopy and delayed childbearing. The use of hormones in menopausal women and obesity may be responsible for the occurrence of endometriosis in postmenopausal age. Most women with endometriosis present some or all of four major problems: infertility, secondary dysmenorrhea, dyspareunia and pelvic pain, but many women are asymptomatic. The intensity of the pain is poorly correlated with the 
actual extend of the disease and the various local biochemical factors and the local action of activated mast cells may be responsible for this symptom. In rare cases with ovarian endometriosis, acute abdomen because of rupture of ovarian cystic masses and ascites raise the suspicion of malignancy (Henkel et al, 1999).

\section{Serum markers in endometriosis}

Levels of CA-125 may be elevated in the serum and peritoneal fluid of women with endometriosis and the concentration of serum CA-125 usually correlate with the severity and the clinical course of the disease. The sensitivity of the serum test is low for the screening of general population, but sensitive in the monitoring the response to hormonal treatment of women with endometriosis. Antiendometrial antibodies are found in $83 \%$ of women with confirmed endometriosis and the titers low after hormonal treatment and a good response.

\section{Etiologic factors of endometriosis}

Genetic, hormonal, immunological, and environmental and angiogenetic factors are implicated in the pathogenesis of endometriosis. Heritable factors are important and there is strong evidence that a large proportion of female relatives are affected by the disease by a severe form of the disease. The relative risk of endometriosis in a first-degree relative is 7.2 and there are high concordance rates for the presence of endometriosis in monozygotic but not dizygotic twins (Kennedy, 1997, Kennedy, 1999). The finding of endometriosis in reproductive years and the hormone dependent response of endometrium implicates hormonal factors in the pathogenesis of endometriosis. The ovarian function is considered an important factor. The concentrations of microenvironmental steroid hormone are high, measuring 1000-fold higher in the ovarian follicles than the plasma .The peritoneal fluid is derived mainly by peri-follicular and corpus luteum exudates and presents high steroid hormone levels. It is estimated that the concentration of peritoneal estradiol during follicular phase increases progressively and reaches after the ovulation levels 100 -fold higher that the plasma concentrations while the progesterone peritoneal levels are low in the follicular and increase rapidly after ovulation. There is evidence that the peritoneal fluid in women with endometriosis contain multiple types of free floating cells such as macrophages, leucocytes, lymphocytes, eosinophils and mast cells in greater numbers than in healthy women (Arid, 1997). Macrophages secret a high number of growth factors such as epidermal and transforming growth factor, fibroblast growth factor, interleukins 1,6 and 8, tumor necrosis factor-a. These factors play a role in the growth and regulation of the endometriotic foci. There is also reported a reduced T-mediated cytotoxicity to autologous endometrial cells and a decreased lymphocyte stimulation response to autologous endometrial antigens in patients with endometriosis. This findings support the theory that certain cell-mediated immune mechanisms limiting the growth of endometriotic implants may be impaired. Cyclo-0xygenase-2, involved in the synthesis of prostaglandins E2 may also play a role in the development of endometriosis. In the peritoneal fluid of women with endometriosis, potent angiogenetic growth factors were observed produced from peritoneal fluid macrophages, which probably are important in the development of endometriosis (Haney, 1991). 


\section{Theories about the histogenesis of endometriosis}

The many theories proffered to explain the histogenesis of endometriosis may be divided in

a. theories that propose the transplantation on endometrium in extra-uterine site,

b. theories that favor the induction of mesenchyme to produce endometriotic tissues under the influence of various factors and

c. theories proposing that endometriosis arise on sites of metaplastic change of the coelomic peritoneum.

The theory of transplantation is the most widely accepted. According to this theory the menstrual flow products flow in a retrograde fashion through the fallopian tubes into the peritoneal cavity. The endometrial products implant, grow and produce tissues with morphology of endometrial glands and stroma. Laparoscopic studies show that during menstruation there is blood in the peritoneal cavity of most women and the retrograde menstrual flow is indeed a usual phenomenon which explains the endometriotic implantation in the ovary and the uterosacral ligaments. Sigmoid colon partly forms a shield and entraps the retrograde menstrual flow products and this fact explains the finding that endometriosis is observed mainly in the left ovary. Also the uterine position is important for the development of endometriosis which is observed anteriorly in patients with anterior uteri. Experimental findings support the transplantation theory and suggest that endometriosis develop in two distinct phases. In the first phase the uterine products are invasive and ovarian hormone -dependent and in the second phase express endogenous estrogen biosynthesis. By the first month after inoculation of primate models with menstrual endometrial tissues these elements attach, grow and invade through the peritoneal mesothelial covering and express estrogen receptor-beta, although aromatase activity is expressed later (Fazleabas et al, 2002 ). Other routes of implantation such as vascular and/or lymphatic spread and direct implantation are reported and explain the development of endometriosis in distant and unusual places such as lung or lymph nodes.

The metaplastic theory proposes that the peritoneum adjacent to ovaries is a multipontential tissue which may obtain characteristics of endometrial tissue (Lauchlan 1972, Lauchlan, 1994). This theory is supported by the observation that endometriosis may develop in cases where no retrograde menstrual flow is possible such as in Rokitansky-Kuster-Hauser syndrome (Acien et al, 1988), in young girls and in men. Finally, according to the induction theory which adds information to transplantation theory, the endometriotic glands and stroma develop de novo from the host tissues, stimulated by the transplanted endometrium.

Most pathologists favor a combination of the metastatic and metaplastic theories and support the theory that endometriosis represents a polygenetic disorder, with alterations in multiple biological pathways (Donnez et al, 2002, Sasson \& Taylor, 2008) leading to a metaplastic process under the irritating effect of endometrial tissue shed during retrograde menstrual flow.

\section{The theory of the secondary Mullerian system}

Lauchlan (1972) first used this term to describe the propensity of the peritoneum which covers the ovary and the lower peritoneal cavity to Mullerian differentiation. The mesothelial covering and the sub-mesothelial stroma of peritoneum exhibit a full spectrum 
of Mullerian differentiation from benign to malignant .Many lesions of secondary Mullerian system are described, which are benign, such as endosapingiosis, endocervicosis, of low grade of malignancy, serous and mucinous and malignant, described as extraovarian peritoneal serous and mucinous carcinomas. The sub-mesothelial stroma gives rise to peritoneal leiomyomatosis and may undergo decidual reaction. Amongst the lesions of secondary Mullerian system are also classed lesions of the retroperitoneal lymph node such as Mullerian inclusions, leiomyomatosis and decidual reaction. Endometriosis is considered as the main lesion of the secondary Mullerian system (Clement \& Young, 2008) It has been postulated that the peritoneum of the lower abdominal cavity retains the ability to differentiate to Mullerian tissues due to the proximity to the coelomic epithelium from which the primary Mullerian system is derived. Lauchlan (1994) proposes that the development of peritoneal Mullerian lesions may be secondary to the proximity of pelvic peritoneum to tubal fibria, and the exposure of the peritoneal surfaces to external agents, such as talk, that stimulate the peritoneal Mullerian differentiation.

\section{Histological features of the secondary Mullerian system}

The secondary Mullerian system consists of the covering mesothelial layer and the submesothelial mesenchyme, on and adjacent to the ovaries, the pelvic peritoneum, the omentum and the retroperitoneal lymph nodes. The most common type of epithelial differentiation is a tubal-like epithelium, with ciliated cells. In the omentum and the ovaries cystic inclusions of tubal type epithelium are often observed intermingled with endometrialtype epithelium. Differentiations to mucinous, transitional and squamous type epithelial lesions, benign and malignant are also described. The sub-mesothelial stroma retains the ability to differentiate towards endometrial stroma and smooth muscle tissue and to undergo decidual reaction.

\section{Elevated serum CA- 125 levels may be expressed in endometriosis}

CA 125 is an antigenic determinant recognized by the monoclonal antibody OC125 against an ovarian cancer cell line. The serum CA125 levels are increased in women with benign conditions such as endometriosis, pelvic inflammatory disease and pregnancy. Ca125 is detected by immunohistochemical methods in fetal coelomic epithelium, fetal Mullerian ducts and Mullerian-derived epithelia in the adult. In the fetus, CA125 is detected in the peritoneum, ovaries and Mullerian ducts after 15 weeks gestation and after the $23^{\text {rd }}$ week it is strongly expressed in endometrium, tubes and cervical tissues.CA125 is considered a differentiation antigen associated with fetal coelomic tissues, derived from embryonic mesoderm Lesions derived from embryonic coelomic tissues either the primary or the secondary Mullerian systems, such as endometriosis, retain the ability to express CA 125.

\section{Common locations of endometriosis}

Most patients with endometriosis have multiple sites of involvement. Endometriosis is located in uterosacral ligaments $(63 \%)$, ovaries (56\%), ovarian fossae $(33 \%)$ anterior vesicle pouch $(22 \%)$ pouch of Douglas $(19 \%)$, intestines, fallopian tube and uterus $(5 \%)$ There is variation of the distribution among the various series, depending on the diagnostic criteria used, histological or clinical. When histological criteria are used, the ovaries are the most 
common site (36\%) the fallopian tubes, uterus and cul-de-sac account for $6-14 \%$ of biopsies, and the uterosacral ligaments account only for $2 \%$ of specimens examined. On a clinical basis, $5-12 \%$ of women present extrapelvic endometriosis and in descending order in the sigmoid, the appendix, and omentum, on operative scars and in inguinal region. Rarely endometriosis is observed in distant sites such as lungs, brain, bones and skin (Jenkins et al, 1986).

\section{The effect of pregnancy on endometriosis}

Endometriosis during pregnancy presents a variable behavior among different patients and a variable behavior in the same patient during different pregnancies. The endometriotic lesions usually shrink and an amelioration of the symptoms is observed. In early pregnancy the lesions may enlarge and bleed. The endometriotic stromal decidual transformation during pregnancy may cause softening and rupture of the lesion causing acute abdomen and hemoperitoneum.

\section{Eendometriosis may cause a Meigs-like syndrome}

In patients with extensive abdominal endometriosis, a sero-sanguinous ascites may occur and in one third of these patients a right pleural effusion may be observed. In these cases, there is also present an ovarian lesion caused by endometriosis, and the whole clinical picture is consistent with malignancy. The exact mechanism of the development of ascites and the pleural effusion is not clear, and as the most probable cause is considered the stimulation of the mesothelial layer by the endometriosis to produce ascites.

\section{Gross features of endometriosis}

The location and the age of the endometriotic lesion and the patients' age affect the morphological appearance of endometriosis and may lead to diagnostic difficulties. Gross appearance of endometriotic lesions is affected by their age and this is reflected by the various colors they present. Red color characterizes early lesions and yellow-red color reflect the breakdown of blood products. These lesions eventually progress into old or advanced lesions presenting black color. Hemosiderin is indicated by a yellowish color and occasionally white lesions may be observed indicating the presence of fibrosis. It is possible that the same patient presents endometriotic foci in various stages of development. The size of the lesions varies as well. In early stages blister-like blebs are observed measuring 0.2-0.3 $\mathrm{cms}$ in diameter, corresponding to the early red lesion observed mainly in adolescents .As the lesions age, they may enlarge up to $1 \mathrm{cms}$ in diameter and are pigmented, bluish-red, black and eventually white and puckered because of fibrosis. Endometriotic foci are frequently associated with adhesions. The older the patient the more fibrotic the endometriotic lesion is and eventually it atrophies with obliteration of its components Endometriotic lesions become grossly cystic only in the ovaries reaching a diameter up to 15 cms. Ovarian endometriotic cysts present a fibrous wall of various thickness and are filled by chocolate-like contend. The interior surface may be smooth or shaggy. Rarely, endometriosis obtains the form of polypoid masses projecting from serosal surfaces and is referred as polypoid endometriosis. 


\section{Microscopic features of endometriosis}

Typically endometriosis in women of reproductive age presents histologically as one or more endometrioid glands surrounded by stromal cells, resembling the endometrial stromal cells of the proliferative phase. The glandular epithelium is one layer thick with cuboidal or tall cells and eosinophilic cytoplasm. Nuclei are ovoid with vertical orientation and very rare mitoses. The whole picture is usually consistent with inactive or irregular proliferative endometrium, although typical proliferative or secretory changes may be observed. Cilia may be observed as well. Stromal cells are supported by a delicate reticulin network in which hyperemic small vessels may be observed. In the case of exogenous administration of progestins, cyclically functionic endometriosis or pregnancy, a stromal decidual reaction may be observed. A diffuse infiltration of histiocytes is usually observed. The histiocytes convert the red blood cells into glucolipid and brown pigment (pseudoxanthoma cells).The pigment is usual a ceroid such as lipofuscin and to a lesser extend hemosiderin .The amount of the pigment increases with the age of the lesion. Inflammatory cells may be present and a small component of smooth muscle cells especially in the wall of endometrioid cysts may be observed. Not all the above described elements are easily identified in endometriosis. Especially in the cases of ovarian endometrioid cysts the lesion appears to be composed of stroma, with fibrosis, lined by hemosiderin-laden macrophages. Many histological sections may be necessary to identify the glandular component of endometriosis. One must keep in mind that macrophages may be connected with hemorrhagic follicles or corpora lutea and only the presence of glandular epithelium or luteinized cells is diagnostic. The degree of cyclic changes of the glandular component depend on the amount of fibrous tissue, the amount of stroma round the glands, the degree of vascularity and the steroid receptor content Endometriosis in places with native smooth muscle component may induce marked hypertrophy identical to that observed in cases of adenomyosis that leads to creation of adenomyomata or adenomyomatous nodules (Anaf et al, 2000).

\section{Common problems in the differential diagnosis of endometriosis}

In the ovary, the presence of hemorrhagic follicle cysts or cystic corpora lutea may cause diagnostic problems, although the presence of granular layer cells or luteinized cells aid to the diagnosis. Serosal inclusion cysts lined by serous cells are diagnosed as such because of the absence of a stromal component and associated alterations. Rete ovarii present a characteristic ramifying pattern and absence of stromal cells and hemorrhage. Mesonephric and paramesonephric remnants are surrounded by smooth muscle and are lined with low cuboidal epithelium. Dermoid cysts may be lined by macrophages and granulation tissue but in multiple sections squamous cells and hair fragments will be observed. It must be noted that there are cases that the laparoscopic picture is diagnostic but the typical picture of endometriosis cannot be established and only stromal cells and other changes such as hemorrhage and macrophages are observed. In these cases the most appropriate diagnosis is that the lesion is "compatible with endometriosis".

\section{Metaplastic and hyperplastic glandular changes observed in endometriosis}

The glandular cells of endometriosis may present metaplastic changes such as ciliated, eosinophilic, clear cell and rarely squamous, transitional and rarely mucinous metaplasia 
usually of endocervical type. It is reported that in cases with extensive metaplastic changes in endometriosis an association with an ovarian epithelial tumor is observed. Glandular epithelium may present hyperplastic changes due to endogenous or exogenous hormonal action resembling hyperplastic endometrial changes from simple cystic to complex atypical hyperplasia. In cases of ovarian endometrioid adenocarcinoma remnants of endometriosis with hyperplastic changes are observed. Metaplastic changes are observed in the endometriotic stroma as well, of smooth muscle type. Concomitant hyperplastic changes may create endomyometriotic nodules or uterus-like masses, in the ovary, broad ligament, the bowel and lymph nodes.

\section{Histological characteristics of stromal endometriosis}

The presence of stromal elements in the absence of glandular epithelium in foci of endometriosis constitutes the stromal endometriosis. This is encountered in the ovary, in the cervix and the peritoneum and usually is associated with a focus of typical endometriosis.

\section{Hormonal receptor status in endometriosis}

In endometriotic glands and stroma, estrogen and progesterone receptors are present in lower concentrations and without the cyclical variation observed in the normal endometrium. There is not correlation between the hormonal receptor status of endometriosis and the severity of symptoms.

\section{Classification of endometriosis}

The American Society for Reproductive Medicine proposed a classification of endometriosis with the aim to predict the chance of conception based on the severity of the disease. This system proposes a point score that assesses: the extend of endometriosis among organs and within an organ, scoring the superficial versus the invasive disease, the size of endometriomas, the development of endometriosis uni-or bilaterally in ovaries , and the type of adhesions. The cumulative score results in a stage designation of the disease, from minimal (stage 1) to extensive (stage 4). The reproducibility of the classification system is limited, according the operative method used and the surgeon's experience (Hoeger \& Guzick, 1997).

\section{Morphology and histogenesis of Liesegang rings}

These rings are acellular eosinophilic structures composed from colloidal solutions, encountered in endometrioid cysts, in areas of inflammation and necrosis. On histological examination, these structures look like parasites and foreign material and may be recognized by their association to endometriosis and lack of staining by specific histochemical methods.

\section{Histological features of pseudoxanthomatous salpingitis}

This is a form of tubal inflammation characterized histologically by the presence of histiocytes filled with lipofuscin and hemosiderin. In multiple sections there is always a typical focus of endometriosis of the fallopian tube and / or the ovary. 


\section{Histological features of necrotic pseudoxanthomatous nodules}

These lesions occur to postmenopausal women and are multiple nodules usually attached to peritoneum or floating free in the peritoneal cavity. They consist of a central fibrotic or hyalinized core with central necrosis surrounded by lipid laden histiocytes. Occasionally multiple sections will reveal remnants of endometriotic foci, and typical ovarian endometriosis co-exists.

\section{Histological features of superficial and deep cervical endometriosis}

Superficial cervical endometriosis is observed in the lamina propria of ectocervix as a solitary or multiple hemorrhagic nodules (endovervix is rarely involved by endometriosis). The predisposing factor is cervical trauma and implantation of endometrioid tissue after surgery. Deep cervical endometriosis is created by the extension of a cul-de-sac lesion and is observed in widespread pelvic endometriosis. Similar mechanisms underlie superficial and deep vaginal endometriosis as well.

\section{Histology of the three types of tubal endometriosis}

Tubal endometriosis may occur as: a direct extension of endometrial tissue with replacement of tubal mucosa, as a growth of endometrial tissue into the lumen with total occlusion causing infertility and associated with tubal pregnancy and, as postsalpingectomy endometriosis, following tubal ligation.

\section{Histological features of spontaneous umbilical endometriosis}

Endometriosis of the umbilicus in the absence of previous caesarian section or hysterectomy is described as spontaneous endometriosis and it is caused by lymphatic dissemination of endometrial tissue, through lymphatic vessels extending between pelvis and umbilicus. Umbilical endometriosis may recur after surgical resection (Chen, 1998).

\section{Histological features of pleuropulmonary endometriosis}

There are 21 cases of documented pleural endometriosis involving exclusively the right side of thorax and 17 cases of pulmonary endometriosis. These cases were associated with abdominal endometriosis in one third of the cases and with uterine operations in half of the cases. The development of pulmonary endometriosis suggests an embolic origin of the endometrial implants, while the pleural involvement is explained by the passage of endometrial implants through diaphragmatic lymphatics or defects.

\section{Histological features of endometriosis of the nervous system}

There are about 30 cases reported of endometriosis of the sciatic nerve, one case of endometriosis of the lumbar spinal cord and one case of cerebral endometriomas. These cases developed probably by "metastatic" implantation of endometrial tissue, through the perineural lymphatics (Abrao et al, 1999, Ichida et al, 1993). 


\section{Endometriosis in male patients}

There are a few reports of endometriosis that develops in male patients under hormonal therapy for prostatic carcinoma. The usual sites that endometriosis develops are: the urinary bladder, the prostate, the para-testicular region and the abdominal wall.

\section{Histological features of the peritoneal decidual reaction and the peritoneal leiomyomatosis}

Peritoneal decidual reaction is an incidental finding, usually discovered during a caesarian section or postpartum tubal ligation, as small whitish or yellow peritoneal nodules, mimicking metastatic disease. Histological examination shows an extensive decidual reaction of the sub-mesothelial mesenchymal cells. This reaction may also be observed at the omentum, appendix and uterine ligaments. Due to the presence of hyperemic vessels in these lesions, the development of hemoperitoneum during labor may be a rare complication Peritoneal leiomyomatosis presents as multiple small white nodules in the peritoneum and arise from smooth muscle-cell metaplasia of the sub-mesothelial mesenchymal cells. This is a self-limiting disease successfully treated with GHRH antagonists. There is no evidence that these two conditions are related to endometriosis, but they probably represent lesions of the secondary Mullerian system (Clement, 1995).

\section{Neoplasms related to endometriosis}

The exact rate of neoplastic transformation of endometriosis is not known because of the fact that the neoplasm obliterates the endometriotic features of the underlying lesion. Neoplastic transformation of both the glandular and the stroma component of endometriosis may occur. There are benign and malignant lesions that may develop in endometriotic foci. Tumor-like benign lesions in the form of nodules or even larger, uterus-like masses are reported. Histological examination showed hyperplastic chances of the glandular component of endometriosis and extensive leiomyomatous metaplasia of the stromal component. These lesions may be described as endometrioid adenomas or endometrioid cystadenomas. Malignant transformation of the glandular component presents the pathological features of an endometrioid adenocarcinoma. In $75 \%$ of cases the malignant transformation of the glandular component arises in the ovaries. Ovarian endometrioid and clear cell adenocarcinomas are strongly related to ovarian endometriosis and atypical endometriotic lesions were observed in $60 \%$ of those carcinomas. Endometriosis -associated ovarian adenocarcinomas are well differentiated tumors in stage I and have a better prognosis that the other ovarian epithelial carcinomas. There are strict criteria for establishing the origin of an adenocarcinoma from endometriosis: Both elements must coexist, atypical hyperplastic and dysplastic changes of endometriosis must be observed and $t$ no extension of carcinoma from another source is established. Extra ovarian sites where malignant transformation of endometriosis is reported are the rectovaginal septum and the colon (Benoit et al, 2006). Malignant transformation of the stromal component of the endometriosis gives rise to stromal sarcomas similar to uterine stromal tumors (Dogan et al, 2006, Han, 1998). In the table below, tumor-like conditions and tumors, benign and malignant, associated with endometriosis are presented. This classification is based on the WHO classification of Ovarian Tumors (IARC Press, Lyon 2003) modified to contain the tumor-like conditions and premalignant changes. 


\begin{tabular}{|c|} 
Tumor-like Conditions \\
Polypoid endometriosis \\
Benign Tumors \\
Adenoma endometrioid \\
Adenofibroma endometrioid \\
Cystadenofibroma endometrioid \\
Cystadenoma endometrioid \\
Premalignant changes \\
Atypical endometriosis \\
Bordeline tumors \\
Endometrioid cystic Adenofibroma \\
Endometrioid Cystadenofibroma \\
Malignant tumors \\
Endometrioid adenocarcinoma \\
With squamous elements \\
With clear cell elements \\
Stromal sarcoma \\
Low grade \\
High grade \\
Malignant tumors with mixed components \\
Adenosarcoma \\
Carcinosarcoma
\end{tabular}

Table 1. Tumor-like conditions and tumors associated with endometriosis

\section{Pathogenesis of malignant transformation of endometriosis}

The molecular pathology studies of both atypical and malignant glandular epithelium of endometriosis have shown aneuploid DNA, in contrast to adjacent benign epithelium which is diploid, and a loss of heterozygosity on the arm of chromosome 12 or X-chromosome inactivation, consistent with a common lineage. Aneuploidy of chromosome 17, implicated in the genesis of ovarian cancer, was discovered in about $65 \%$ of the endometrioid cells (Jiang et al, 1998). There is evidence that malignant transformation of endometriosis is a multistep pathway involving somatic genetic changes and changes in hormone receptor status (Hompes \& Mijatovitc, 2007).

In conclusion, endometriosis is a chronic gynecological disease that results in severe morbidity, including chronic pain and infertility. The pathogenesis of the condition is multifactorial and various factors, genetic, immunological, environmental are implicated Recent data provide evidence that there is an endometrial stem/progenitor cell capable of establishing endometriotic implants .The functions of this stem cell may represent the primary defect in the endometriosis pathway. Current research uses the endometrial stemcell system as a model to study the molecular biology regulating endometriosis (Sasson \& Taylor, 2007).

\section{References}

Abrao MS, Podgaec S, Carvalho FM, Pinotti JA (1999). Endometriosis in the presacral nerve. Int J Gynecol Obstet 64:173-175. 
Acien P, Lloret M, Chehab H (1988). Endometriosis in a patient with Rokitansky- KusterHauser syndrome. Gynecol Obstet Invest 25:70-72.

Allaire C. Endometriosis and infertility - a review (2006). J Reprod Med 51:164-8.

Anaf V, Chapron C, El Nakadi I, De Moor V, Simonart T, Noel JC (2006). Pain, mast cells, and nerves in peritoneal, ovarian, and deep infiltrating endometriosis. Fertil Steril 86:1336-43.

Anaf V, Simon P, Fayt I, Noel J (2000). Smooth muscles are frequent components of endometriotic lesions. Hum Reprod 15:767-71.

Arid A, Oral E (1997). The peritoneal environment in endometriosis. In: Diamond MP, Osteen KG, eds. Endometrium and Endometriosis. Oxford: Blackwell Science; 161-73.

Benoit L, Arnould L, Cheynel N, et al (2006). Malignant extraovarian endometriosis: a review. EJSO 32:6-11.

Chen TH(1998). Umbilical endometriosis. Eur J Plast Surg 21:51-2.

Clement PB (1995). Reactive tumor-like lesions of the peritoneum. Am J Clin Pathol 103:673-6.

Clement PB and Young RH (2008). Atlas of Gynecologic Surgical Pathology, 2nd Ed. SaundersElsevier ISBN 978-1-4160-2972-4, China.

Cramer DW, Missmer SA (2002). The epidemiology of endometriosis. In: Yoshinaga, Parrott EC, eds. Endometriosis: Emerging Research and Intervention Strategies. New York: New York Academy of Sciences, 11-22.

Dogan S. Agic A, Eilers W, Finas D, Diedrich K, Hornung D (2006). Endometriosis and risk of malignancy. Geburt Frauenheil 66:739-44.

Donnez J, Van Langendonckt A, Casanas-Roux F, et al (2002). Current thinking on the pathogenesis of endometriosis. Gynecol Obstet Invest 54(Suppl):52-62.

Fazleabas AT, Brudney A, Gurates B, Chai D, Bulun S (2002). A modified baboon model for endometriosis. In: Yoshinaga K, Parrott EC, eds. Endometriosis: Emerging Research and Intervention Strategies. New York: New York Academy of Sciences, 308-17.

Han AC, Hovenden S. Rosenblum NG, Salazar H (1998). Adenocarcinoma arising in extragonadal endometriosis: an immunohistochemical study. Cancer 83:1 163-9.

Haney AF. The pathogenesis and etiology of endometriosis (1991). In: Thomas EJ, Rock JA, eds. Modern Approaches to Endometriosis. Dordrecht: Kluwer Academic, 113-28.

Henkel A, Christensen B, Schindler AE (1999). Endometriosis: a clinically malignant disease. Eur J Obstet Gynecol Reprod Biol 82:209-11.

Hoeger KM, Guzick DS (1997). Classification of endometriosis. Obstet Gynecol Clin North Am 24:347-59.

Hompes PGA, Mijatovic V (2007). Endometriosis: the way forward. Gynecol Endocrinol 23:512.

Ichida M, Gomi A, Hiranouchi N, et al (1993). A case of cerebral endometriosis causing catamenial epilepsy. Neurology 43:2708-9.

Jenkins S, Olive DL, Haney AF (1986). Endometriosis: pathogenetic implications of the anatomic distribution. Obstet Gynecol 67:335-8.

Jiang XX, Morland SJ, Hitchcock A, Thomas EJ, Campbell IG (1998). Allelotypi of endometriosis with adjacent ovarian carcinoma reveals evidence of a common lineage. Cancer Research 58:1707-12.

Kennedy S (1997). Is there a genetic basis to endometriosis? Semin Reprod Endocrinol 15:30918.

Kennedy S (1999). The genetics of endometriosis. Eur J Obstet Gynecol Reprod Biol 82:129-33. 
Knapp VJ (1999). How old is endometriosis? Late 17th- and 18th-century European descriptions of the disease. Fertil Steril Jul;72(1):10-4.

Lauchlan SC (1972). The secondary Mullerian system. Obstet Gynecol Surv 27(3):133-46

Lauchlan SC (1994). The secondary Mullerian system revisited. Int J Gynecol Pathol 13(1):73-9

Sasson IE,Taylor HS (2008). Stem cells and the pathogenesis of endometriosis. Ann. NY Acad. Sci. 1127:106-115 


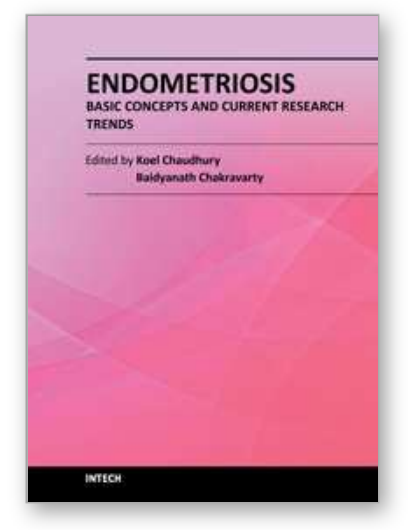

\author{
Endometriosis - Basic Concepts and Current Research Trends \\ Edited by Prof. Koel Chaudhury
}

ISBN 978-953-51-0524-4

Hard cover, 490 pages

Publisher InTech

Published online 09, May, 2012

Published in print edition May, 2012

This book provides an insight into the emerging trends in pathogenesis, diagnosis and management of endometriosis. Key features of the book include overviews of endometriosis; endometrial angiogenesis, stem cells involvement, immunological and hormonal aspects related to the disease pathogenesis; recent research reports on infertility, endometrial receptivity, ovarian cancer and altered gene expression associated with endometriosis; various predictive markers, and imaging modalities including MRI and ultrasound for efficient diagnosis; as well as current non-hormonal and hormonal treatment strategies This book is expected to be a valuable resource for clinicians, scientists and students who would like to have an improved understanding of endometriosis and also appreciate recent research trends associated with this disease.

\title{
How to reference
}

In order to correctly reference this scholarly work, feel free to copy and paste the following:

Agatha Kondi-Pafitis (2012). Pathological Aspects of Endometriosis, Endometriosis - Basic Concepts and Current Research Trends, Prof. Koel Chaudhury (Ed.), ISBN: 978-953-51-0524-4, InTech, Available from: http://www.intechopen.com/books/endometriosis-basic-concepts-and-current-research-trends/pathologicalaspects-of-endometriosis

\section{INTECH}

open science | open minds

\author{
InTech Europe \\ University Campus STeP Ri \\ Slavka Krautzeka 83/A \\ 51000 Rijeka, Croatia \\ Phone: +385 (51) 770447 \\ Fax: +385 (51) 686166 \\ www.intechopen.com
}

\author{
InTech China \\ Unit 405, Office Block, Hotel Equatorial Shanghai \\ No.65, Yan An Road (West), Shanghai, 200040, China \\ 中国上海市延安西路65号上海国际贵都大饭店办公楼405单元 \\ Phone: +86-21-62489820 \\ Fax: +86-21-62489821
}


(C) 2012 The Author(s). Licensee IntechOpen. This is an open access article distributed under the terms of the Creative Commons Attribution 3.0 License, which permits unrestricted use, distribution, and reproduction in any medium, provided the original work is properly cited. 